\title{
Optimization of hematopoietic induction from induced pluripotent cells
}

\begin{abstract}
Acute myeloid leukemia is the most aggressive form of leukemia that results in immature hematopoietic stem cells (HSCs) that are unable to undergo normal hematopoiesis. Currently, the literature has shown that one can derive HSCs from induced pluripotent stem cells (iPSCs) by co-culturing iPSCs with OP9 stromal cells.1 While this is an effective method of generating HSCs, the mechanism behind this induction of hematopoiesis is not fully understood yet. It remains unclear whether direct contact of OP9 cells is necessary to induce hematopoietic differentiation or if simply the proteins or transcription factors that OP9 cells secrete are sufficient. Our review examines the current literature on the mechanism behind hematopoiesis involving the OP9 co-culturing methodology and the isolation of molecular components that may be responsible for induced hematopoiesis. Significant OP9 excretions that may play a role in hematopoietic induction are: Lhx2, Hoxb4, Bmi-1, IL-7, and AML1-ETO Fusion Protein. In this paper we explore the characteristics of these proteins and their potential applications towards the optimization of hematopoietic induction.
\end{abstract}

Keywords: acute myeloid leukemia, ipsc, hematopoiesis, hematopoietic stem cells, op9 coculture, stem cells, cell culture
Volume 3 Issue I - 2017

\author{
Michael Amedeo, Prableen K Chowdhary, \\ Aria Jalalian, Wei Chen Lai, Amil Sahai, \\ George Thomas, Akhil Uppalapati, Farah \\ Vejzagic, Nam Sun Wang \\ Department of Chemical and Biomolecular Engineering, \\ University of Maryland, USA
}

\begin{abstract}
Correspondence: Nam Sun Wang, Department of Chemical and Biomolecular Engineering, University of Maryland, College Park, MD 20742, USA, Tel 301-405-1910, Fax 301-405-0523,
\end{abstract} Email nsw@umd.edu

Received: September 13,2017| Published: October 06, 2017
Abbreviations: iPSC, induced pluripotent stem cells; HSC, hematopoietic stem cells; IL, interleukin; GM-CSF, granulocyte-macrophage colony-stimulating factor

\section{Introduction}

Recent literature has demonstrated the possibility of deriving hematopoietic stems cells from induced pluripotent stem cells by coculturing the iPSCs with OP9 stromal cells. Although HSCs can be derived from iPSCs, researchers continue to investigate the molecular factors that play important roles in this process. If HSCs can be effectively developed with improved yields, the medical community may potentially reduce the number of blood transfusions that leukemia patients would have to undergo. An overall review of the current literature will be critical in further understanding hematopoiesis and the engraftment potential of iPSCs in leukemia patients.

\section{Discussion}

\section{iPSC differentiation techniques and hematopoiesis}

Hematopoiesis is the process by which the cellular components of blood are formed- usually during development. In vertebrates, there are two documented waves of hematopoiesis; the primitive wave and the definitive wave. ${ }^{2}$ The first wave, the primitive wave, occurs during early embryonic development and gives rise to the erythrocytes and macrophages. This wave is integral to development, as it provides the red blood cells necessary for tissue oxygenation throughout the rest of development. This stage is transient, and later gives rise to the definitive wave of hematopoiesis. Within the definitive wave, which continues much throughout adulthood, the entire lineage of blood cells are able to be formed, including red blood cells, white blood cells, platelets. Among the various types of cells, multipotent HSCs are generated and are harbored mostly within the bone marrow. ${ }^{3}$
In 2006, researchers at Kyoto University in Japan identified a specialized protocol that would induce adult cells to be "reprogrammed" into cells that were pluripotent and could be differentiated into virtually any cell type. ${ }^{4}$ The discovery of induced pluripotent stem cells (iPSCs) led to new avenues of investigation in the innovative field of regenerative medicine, with the potential to provide personalized medical therapy and circumvent issues such as graft versus host disease. These cells also managed to avoid some of the ethical issues that belie regular stem cells, as they were developed from each individual patient's own cells - a further avenue exploring personalized stem cell therapy.

In a 2011 study led by Salvagiotto et al., ${ }^{5}$ it was shown that a single matrix protein in concert with hypoxic conditions were sufficient to efficiently generate pluripotent hematopoietic stem cell progenitors in vitro. Nine lines of iPSC cells were tested to examine which condition would provide the most robust amount of clinical grade progenitors that were induced from pluripotent cells. Hypoxic conditions were used to mimic an early embryonic environment in which HSC progenitor production is often robust. After initiating the protocol and co-culturing of iPSCs with the matrix protein BMP4-VEGF-bFGF, a $5 \%$ oxygen tension was added in order to mimic hypoxic conditions in the embryo. Hematopoietic progenitors were then identified with flow cytometry to count the CD34+ cells, which is a marker that characterizes progenitor cells. The study concluded that, although the clinical introduction of this protocol would be relatively easy due to a plausible switchover in xenophobic conditions, the protocol must be investigated in further detail to see whether higher yields of hematopoietic progenitors could be achieved.

Co-culturing of OP9 mouse bone marrow (BM) stromal cells with iPSCs is as another efficient method that has generated higher yields of hematopoietic progenitors. ${ }^{6}$ This technique, although typically used to differentiate hematopoietic embryonic stem cells (hESCs), has proven to be effective with iPSCs and can be executed with or without 
exogenous growth factors. ${ }^{6}$ The protocol for differentiation consists of three steps: generation of differentiation through the co-culture of the OP9 BM stromal cells and iPSCs, expansion of multipotent common myeloid progenitors (CMPs), and differentiation of the CMPs. After six days of coculture with OP9 feeders, iPSCs begin to show properties of myeloid progenitors capable of differentiation. Expansion was achieved by dissociating the OP9/iPSC coculture and then placing the cells in non-adherent conditions in which they spontaneously reaggregated. ${ }^{6}$ Cells that expressed the phenotype linCD34+CD43+CD45+ were determined to be multipotent HSCs with differentiation potential as opposed to other similar phenotypic cell markers representing endothelial and mesenchymal stem cells. ${ }^{6}$

OP9 coculture creates a wide spectrum of myeloid progenitors, necessitating isolation of the lin-CD34+CD43+CD45+ cells from the other forms of progenitors produced (erythroid and megakaryocytic). Choi et al. ${ }^{6}$ accomplished this through magnet-activated cell sorting, using anti-human antibodies specific to the erythroid and megakaryocytic progenitors. Differentiation of the obtained CMPs was then achieved through the application of cytokine combinations. Choi et al. ${ }^{6}$ asserted that the optimal combinations for the generation of dendritic cells were granulocyte-macrophage colony-stimulating factor (GM-CSF), interleukin-4(IL-4) and tumor necrosis factor- $\alpha$ (TNF- $\alpha$ ); whereas GM-CSF, transforming growth factor- $\beta_{1}$ and TNF- $\alpha$ were optimal for the generation of LCs. For macrophages, the optimal combination was M-CSF and IL- $\beta$, while G-CSF was best for neutrophils. Finally, IL-3 and IL-5 were optimal for eosinophils. The different types of differentiated cells obtained in this study were established by analyzing their cell morphology via cytospins and their phenotype using flow cytometry. Cell markers allow for characterization and identification of the obtained eosinophils, neutrophils, dendritic cells, Langerhans cells, macrophages and osteoclasts. The success of this method of differentiation yields saclike structures, and in exceptionally efficient cases, undifferentiated colonies. ${ }^{6}$

Choi et al. $^{6}$ utilized a similar methodology using the OP9 coculture, examining iPSCs obtained from human fetal, neonatal, and adult fibroblast tissues compared to different forms of hESCs. In this study, the iPSCs and OP9 cells were cocultured without cytokines. This study also used magnet-activated cell sorting to isolate three subsets of HPCs:

$$
\begin{aligned}
& \text { CD235a/CD41a+CD43+CD45-, } \\
& \text { CD235a/CD41a-CD43+CD45-, and } \\
& \text { CD235a/CD41a-CD43+CD45+ cells. }
\end{aligned}
$$

The colonies of erythroid, granulocyte, erythrocyte, macrophage, megakaryocyte, and macrophage cells were assayed and scored after incubation. This method of inducing hematopoiesis with OP9 has proven successful while requiring relatively little time and resources. ${ }^{6}$ As iPSC coculture with OP9 bone mouse stromal cells is simple and efficient, this methodology for achieving hematopoiesis is consistent in optimizing differentiation for all mentioned iPSCs.

\section{Investigation of relevant op9 protein excretions}

\section{IL-7}

While investigating the roles and functions of many proteins that are involved in hematopoiesis through coculturing with OP9 stromal cells, the interleukin family of proteins stand out the most in terms of recognition, effectiveness, and commonality. Interleukin, according to the National Cancer Institute at the NIH, is defined as "a group of related proteins made by leukocytes and other cells that regulate immune responses. Interleukins made in the laboratory are used as biological response modifiers to boost the immune system in cancer therapy". 7 More specifically, an interleukin is a type of cytokine, which can be added during the hematopoietic process to aid in cell differentiation and proliferation. A study that examined the distinctive roles of a specific interleukin, interleukin-7 (IL-7), found that IL-7 aided in the survival and proliferation of the cells as well as providing differentiation signals in a dose-dependent manner. ${ }^{8}$ In another study observing the roles of interleukins in hematopoietic cell development, researchers found that hemangioblasts, cells with the capacity to differentiate into hematopoietic cells, were regulated and promoted by IL-3. ${ }^{9}$

Interleukins, as examples of cytokines, are mediators of communication between cells. ${ }^{9}$ They regulate cell growth and differentiation, and are even named after leukocytes, since they were believed to originate from leukocytes. The interleukin family encompasses many different types of proteins involved in various types of cell development and signaling processes. A compilation of functions and roles of all the known interleukins shows that, IL-1 is known to aid in differentiation into $\mathrm{T}$ cells and fibroblasts, IL-2 for CD4+ and CD8+ T cells, IL-4 for T and B cells, and IL-6 into hematopoietic cells, etc. ${ }^{10}$ Therefore, interleukins are regulatory proteins that act as cytokines to aid in the process of hematopoiesis cell differentiation. Since interleukins come from different types of cells, particularly in OP9 stromal cells, it is possible to further investigate if interleukins consist of the essential proteins that aid in cell differentiation. Identification of proteins such as interleukins may enhance the current understanding of the roles and functions each family plays during cell differentiation. This can be made possible if interleukins play a role in juxtacrine versus paracrine signaling, which is deserving of investigation and experimentation through future research.

\section{Lhx2}

The transduction of Lhx2 into mesodermal cells derived from embryonic stem cells (ESCs) was found to result in c-Kit+/Sca-1+/ Lineage (KSL) cells in vitro. KSL cells represent an early form of HSCs. Furthermore, transduction of Lhx2 into iPSCs was also found to be effective in generating KSL cells in vitro. Data showed that there is no significant difference in the behavior between iPSC derived HSCs and ESC derived HSCs in vivo. Looking into the Lhx2 protein may be a fruitful avenue of investigation as it may be used in OP9-iPSC interactions in order to induce differentiation into HSCs. If we see that there are no Lhx2 proteins present, this too would be a significant discovery, as it shows that the OP9-iPSC coculture's differentiation into HSC is not dependent on this protein. ${ }^{11}$

\section{Hoxb4}

Traditionally, there has been a problem inducing HSCs to proliferate rather than differentiate. The Hoxb4 transcription factor has been found to be able to cause high levels of HSC expansion ex vivo. Researchers compared GFP transduced murine bone marrow cells to cultures of Hoxb4-transduced cells and found that the cells that were transduced with Hoxb4 resulted in 40-fold net HSC increase. Significantly, these HSCs enhanced regenerative potential in vivo. This transcription factor may play a critical role in HSC 
differentiation and may further elucidate the mechanism by which iPSCs differentiate into HSCs. This is therefore an interesting protein to investigate within the frame of OP9-iPSC interactions; identifying this protein as a component of hematopoiesis may imply that the OP9iPSC coculture system helps maintain HSCs in addition to generating them. ${ }^{12}$

\section{Bmi-1}

HSC formation should occur throughout the lifespan of an organism in order to maintain its viability. One protein that has been shown to be critical to this maintenance of hematopoiesis is Bmi-1, a protein encoded for by the proto-oncogene Bmi-1. Researchers investigated the number of HSCs in prenatal and postnatal mice who were Bmi-1 deficient. Results showed that the mice that were deficient for Bmi-1 had a decreased number of HSCs as compared to the control mice in adulthood; this result, however, was not replicated in the prenatal condition. It was concluded that Bmi-1 is requisite for the creation of self-renewing adult HSCs. A possibility may be that Bmi-1 is present in the coculture in order to help sustain HSC formation from the OP9iPSC coculture, and may be a further avenue of research. ${ }^{13}$

\section{Murine blood cell to HSC transcription factors}

HSCs can also be generated by transient expression of six transcription factors on committed myeloid effector cells and myeloid and lymphoid progenitors. These transcription factors (Run1t1, Hlf, Lmo2, Prdm5, Pbx1, and Zfp37) essentially help run in reverse what the optimization process is attempting to achieve. That is to say that rather from going from a state of higher potency to lower potency, they revert already committed cells to a more potent state. Nevertheless, since they are intimately involved in generating HSCs, the role of these transcription factors in the OP9/iPSC coculturing system warrants further investigation. ${ }^{14}$

\section{AMLI-ETO fusion protein}

Acute myelogenous leukemia-1 (AML1)-ETO fusion protein has been found by researchers to enhance the self-renewal of pluripotent stem cells. Though this protein inhibits the AML1 protein and decreased levels of AML1 has been correlated with a tendency to develop AML, experiments have shown that AML1-ETO does not necessarily increase leukemogenesis. Furthermore, when investigating AML1-ETO, researchers found that it enhanced the growth of stem cells. In the experiment that they conducted, this indicated that cells transduced with this protein had a survival advantage over those that were not. This protein seems to promote the self-renewal of pluripotent cells, which are the targets of many AMLs. Thus, testing for the presence of this protein in the OP9-iPSC coculture is a logical next step, as it has proven to be instrumental to stem cell regeneration and maintenance. ${ }^{15}$

\section{Conclusion}

Previous research has demonstrated the successful differentiation of iPSCs into HSCs through the usage of a promising OP9 coculture methodology. iPSCs may be cocultured with OP9 cells directly or indirectly to determine how differentiation occurs on a cellular level, whether through juxtacrine signaling or paracrine signaling. Proteins that contribute to differentiation may also be investigated via protein quantification and identification methods. By identifying and analyzing relevant proteins, the mechanism behind hematopoiesis may be fully understood and optimized to yield more viable HSCs for future clinical trials. We hope that our review may assist in the ongoing battle against acute myeloid leukemia and inspire future research projects that further investigates this issue.

\section{Acknowledgements}

We would like to thank the Gemstone Honors Program at the University of Maryland as well as Dr. John Kerwin and the Institute for Bioscience and Biotechnology Research (IBBR) at the University of Maryland/NIST, Shady Grove, for their instrumental support and help throughout this process.

\section{Conflict of interest}

The author declares no conflict of interest.

\section{References}

1. Vodyanik MA, Bork JA, Thomson JA, et al. Human embryonic stem ce11-derived CD34+cells: efficient production in the coculture with OP9 stromal cells and analysis of lymphohematopoietic potential. Blood. 2015;105(2):617-626.

2. Orkin SH, Zon LI. Hematopoiesis: an evolving paradigm for stem cell biology. Cell. 2008;132(4):631-644.

3. Jagannathan Bogdan M, Zon LI. Hematopoiesis. Development. 2013;140(12):2463-2467.

4. Takahashi K, Tanabe K, Ohnuki M, et al. Induction of pluripotent stem cells from adult human fibroblasts by defined factors. Cell. 2007;131(5):861-872.

5. Salvagiotto G, Burton S, Daigh CA, et al. A defined, feeder-free, serum-free system to generate in vitro hematopoietic progenitors and differentiated blood cells from hESCs and hiPSCs. PloS one. 2011;6(3):e17829.

6. Choi KD, Yu J, Smuga Otto K, et al. Hematopoietic and endothelial differentiation of human induced pluripotent stem cells. Stem cells. 2009;27(3):559-567.

7. NCI dictionary of cancer terms. National cancer institute.

8. Wang H, Pierce LJ, Spangrude GJ. Distinct roles of IL-7 and stem cell factor in the OP9-DL1 T-cell differentiation culture system. Experimental hematology. 2006;34(12):1730-1740.

9. He WY, Lan Y, Yao HY, et al. Interleukin-3 promotes hemangioblast development in mouse aorta-gonad-mesonephros region. Haematologica. 2009;95(6):875-883.

10. Akdis M, Burgler S, Crameri R, et al. Interleukins, from 1 to 37, and interferon- $\gamma$ : receptors, functions, and roles in diseases. J Allergy Clin Immunol. 2011;127(3):701-721.

11. Kitajima K, Minehata KI, Sakimura K, et al. In vitro generation of HSC-like cells from murine ESCs/iPSCs by enforced expression of LIM-homeobox transcription factor Lhx2. Blood. 2011;117(14):3748-3758.

12. Antonchuk J, Sauvageau G, Humphries RK. HOXB4-induced expansion of adult hematopoietic stem cells ex vivo. Cell. 2002;109(1):39-45.

13. Park IK, Qian D, Kiel M, et al. Bmi-1 is required for maintenance of adult self-renewing haematopoietic stem cells. Nature. 2003;423(6937):302305.

14. Riddell J, Gazit R, Garrison BS, et al. Reprogramming committed murine blood cells to induced hematopoietic stem cells with defined factors. Cell. 2014;157(3):549-564.

15. Mulloy JC, Cammenga J, MacKenzie KL, et al. The AML1-ETO fusion protein promotes the expansion of human hematopoietic stem cells. Blood. 2002;99(1):15-23. 